\title{
A combinatorial non-commutative Hopf algebra of graphs
}

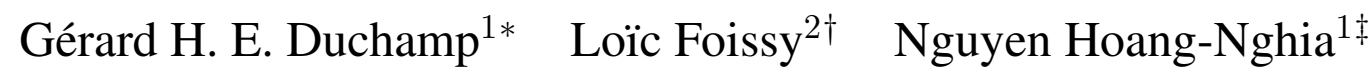 \\ Dominique Manchon ${ }^{3} \quad$ Adrian Tanasa ${ }^{1,4} \|^{\mathrm{T}}$
}

${ }^{1}$ Université Paris 13, Sorbonne Paris Cité, LIPN, Institut Galilée, CNRS UMR 7030, Villetaneuse, France

${ }^{2}$ Université du Littoral, LMPA Joseph Liouville, Calais, France

${ }^{3}$ Université Blaise Pascal - Laboratoire de Mathématiques, CNRS UMR 6620, Aubière, France

${ }^{4}$ Horia Hulubei National Institute for Physics and Nuclear Engineering, Magurele, Romania

received $29^{\text {th }}$ Nov. 2013, accepted $7^{\text {th }}$ May 2014.

A non-commutative, planar, Hopf algebra of planar rooted trees was defined independently by one of the authors in Foissy (2002) and by R. Holtkamp in Holtkamp (2003). In this paper we propose such a non-commutative Hopf algebra for graphs. In order to define a non-commutative product we use a quantum field theoretical (QFT) idea, namely the one of introducing discrete scales on each edge of the graph (which, within the QFT framework, corresponds to energy scales of the associated propagators). Finally, we analyze the associated quadri-coalgebra and codendrifrom structures.

Keywords: noncommutative Hopf algebras, graphs, discrete scales

\section{Introduction}

The Hopf algebra of rooted forests first appeared in the work of A. Dür [11] (and its group of characters, known as the Butcher group, appeared even earlier in the work of J. Butcher in numerical analysis [5]). It has been rediscovered by D. Kreimer in the context of quantum field theory [21], see also [4]. A noncommutative version, using ordered forests of planar trees, has been discovered independently by L. Foissy [13] and R. Holtkamp [19]. Remarkably enough, this Hopf algebra is self-dual. Commutative Hopf algebras of graphs have been introduced and studied by A. Connes and D. Kreimer [7, 8, 9], as a powerful algebraic tool unveiling the combinatorial structure of renormalization.

Inspired by constructive quantum field theory [28], we propose in this article a noncommutative version of a Hopf algebra of graphs, by putting a total order on the set of edges. This can be visualized by putting

\footnotetext{
*Email: ghedelipn. univ-paris13.fr.

†Email: foissy@lmpa.univ-littoral. fr.

‡Email: nguyen. hoang@lipn. univ-paris13.fr.

§Email: manchon@math. univ-bpclermont. fr.

TEmail: adrian.tanasadens-lyon.org.
} 
pairwise distinct decorations on each edge, where the decorations take values in the positive integers (or even in any totally ordered infinite set). We prove that the vector space freely generated by these totally assigned graphs (TAGs) is a Hopf algebra. The product is given by the disjoint union of graphs with the ordinal sum order on the edges (see Formula (3)), and the coproduct is given by Formula (6), involving subgraphs and contracted graphs. In the last section of this paper we analyze in detail the associated quadri-coalgebra and codendrifrom structures.

It is interesting to notice that what we call here TAGs have already been analyzed, from a completely different perspective (the travelling salesman problem), by O. Boruvka, already in 1926 (see [3]). He proved that the shortest spanning tree of such a graph is unique(i). Moreover, the same problem was solved through several simpler explicit constructions by the celebrated Kruskal algorithm [22].

Let us mention here that, throughout this paper, we do not deal with graphs which are necessarily 1particle-irreducible (i. e. bridge-less). Moreover we do not consider in this paper external edges, as it is done in quantum field theory.

\section{Why discrete scales?}

As already announced above, the idea of decorating the edges of a graph with discrete scales comes from quantum field theory, or more precisely from the multi-scale analysis technique used in perturbative and in constructive renormalization (see Vincent Rivasseau's book [28]).

In quantum field theory each edge of a graph is associated with a propagator $C=1 / H$ (which, in elementary particle physics represents a particle). Introducing discrete scales comes to a "slicing" of the propagator

$$
\begin{aligned}
C & =\int_{0}^{\infty} e^{-\alpha H} d \alpha, \sum_{i=0}^{\infty} C^{i} \\
C^{a} & =\int_{M^{-2 a}}^{M^{-2(a-1)}} e^{-\alpha H} d \alpha, C^{0}=\int_{1}^{\infty} e^{-\alpha H} d \alpha .
\end{aligned}
$$

When some discrete integer $a$ is associated to a given edge, this means that the propagator assigned to this edge lies within a given energy scale. One thus introduces more information (replacing graphs by "assigned graph") which yields in turn some refinement of the analysis, as we will explain here.

When integrating over the energy scales of the internal propagators in a Feynman graph in quantum field theory, one obtains the Feynman integral associated to the respective graphs. Usually, these integrals are divergent. This is when renormalization comes in, subtracting (when possible) the divergent parts of these Feynman integrals, in a self-consistent way (see again Vincent Rivasseau's book [28] or any other textbook on renormalization). Nevertheless, these divergences only appear for high energies (the so-called ultraviolet regime (ii), which corresponds, within the multi-scale formalism, to the case when

(i) Note however that within this travelling salesman context the decoration associated to a self-loop (known as a tadpole edge in quantum field theoretical language) is zero, while in our context one can have strictly positive integers associated to such self-loops.

(ii) Divergences for low energies (the infrared regime) can also appear in quantum field theory, but one can deal with this type of divergences in a different way. This lies outside the purpose of this section. 
all the integer scales associated to the internal edges are higher then the edges associated to the external edges (see again Vincent Rivasseau's book [28] for details).

When dealing with this divergence subtraction (the subtraction of the so-called "counterterms"), an important "technical" complication is given by the issue of "overlapping divergences", which is given by overlapping subgraphs which lead, independently, to divergences. This problem is solved in an elegant way within the multi-scale analysis, where all subgraphs leading to divergences are either disjoint or nested.

Let us also emphasize that the multi-scale renormalization technique splits the counterterms into two categories: "useful" and "useless" counterterms (the useful ones being the ones corresponding to subgraphs where all the integer scales associated to the internal edges are higher then the edges associated to the external edges). This refining is not possible without the scale decoration of edges; furthermore, it also solves another major problem of renormalization, the so-called "renormalon problem" (which is an issue when one wants to sum over the contribution of each term in perturbation theory).

This versatile technique of multi-scale analysis was successfully applied for scalar quantum field theory renormalization (see again [28]), the condensed matter case [2],[12],[29], renormalization of scalar quantum field theory on the non-commutative Moyal space (see [16], [17], [18], [30] and [36]) and recently to the renormalization of quantum gravity tensor models [15], [6].

The combinatorics of the multi-scale renormalization was encoded in a Hopf algebraic framework in [20]. As already announced above, the Hopf algebraic setting of [20] is commutative, and the assigned graphs designed there can have equal scale integers for several edges of the same graph.

\section{Non-commutative graph algebra structure}

In this section we define the space of totally assigned graphs (TAG) and a non-commutative algebra structure on this space.

Definition 3.1 $A$ totally ordered scale assignment $\mu$ for a graph $\Gamma$ is a total order on the set $E(\Gamma)$ of edges of $\Gamma$.

It will be convenient to visualize the total order $\mu$ by choosing a compatible labelling, i.e. an injective increasing map from $(E(\Gamma), \mu)$ into $\mathbb{N}^{*}=\{1,2,3, \ldots\}$. There is of course an infinite number of possible labellings. The unique such map with values in $\{1, \ldots, \mid E(\Gamma)]\}$ will be called the standard labelling associated with $\mu$.

Example 3.2 An example of a totally ordered scale assignment with nonstandard labelling is given in Fig. 1 .

Definition 3.3 A totally assigned graph $(T A G)$ is a pair $(\Gamma, \mu)$ formed by a graph $\Gamma$ (not necessarily connected), together with a totally ordered scale assignment $\mu$.

Consider now a field $\mathbb{K}$ of characteristic 0 , and let $\mathcal{H}$ be the $\mathbb{K}$ - vector space freely spanned by TAGs. The product $m$ on $\mathcal{H}$ is given by:

$$
m\left(\left(\Gamma_{1}, \mu\right),\left(\Gamma_{2}, \nu\right)\right)=\left(\Gamma_{1}, \mu\right) \cdot\left(\Gamma_{2}, \nu\right):=\left(\Gamma_{1} \sqcup \Gamma_{2}, \mu \sqcup \nu\right),
$$

where $\Gamma_{1} \sqcup \Gamma_{2}$ is the disjoint union of the two graphs, and where $\mu \sqcup \nu$ is the ordinal sum order, i.e. the unique total order on $E\left(\Gamma_{1}\right) \sqcup E\left(\Gamma_{2}\right)$ which coincides with $\mu$ (resp. $\nu$ ) on $\Gamma_{1}$ (resp. $\Gamma_{2}$ ), and such that 


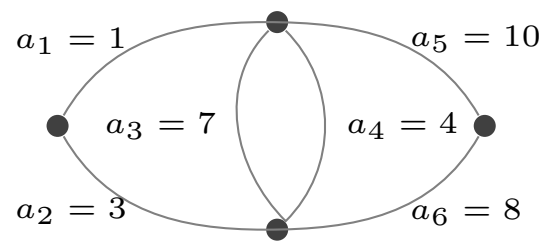

Fig. 1: A graph with a totally ordered scale assignment.

$e_{1}<e_{2}$ for any $e_{1} \in \Gamma_{1}$ and $e_{2} \in \Gamma_{2}$. Although the disjoint union of graphs is commutative, the product is not because the total orders $\mu \sqcup \nu$ and $\nu \sqcup \mu$ are different (see also Remark 3.7 below). Associativity is however obvious. The empty TAG is the empty graph, denoted by $1_{\mathcal{H}}$.

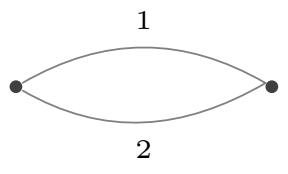

(a) The TAG $\left(\Gamma_{1}, \mu_{1}\right)$.

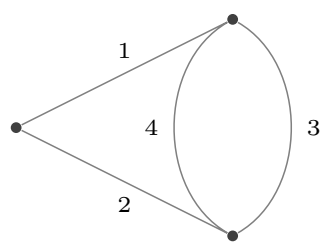

(b) The TAG $\left(\Gamma_{2}, \mu_{2}\right)$.

Fig. 2: Two examples of TAGs.

Example 3.4 Let $\left(\Gamma_{1}, \mu_{1}\right)$ and $\left(\Gamma_{2}, \mu_{2}\right)$ be the two graphs in Fig. 2 .

One has

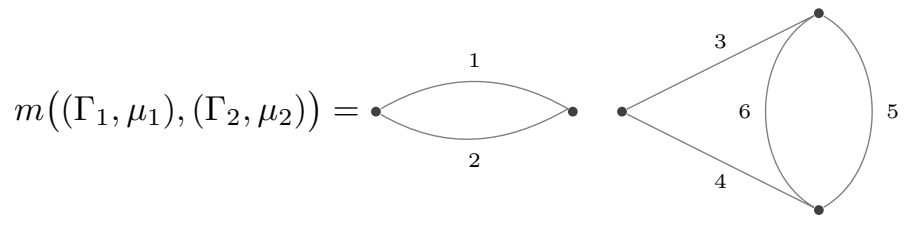

One has:

Proposition $3.5\left(\mathcal{H}, m, 1_{\mathcal{H}}\right)$ is a free associative unitary algebra.

Proof: The only thing which is non-trivial to prove is the freeness of the algebra. Let $G=(\Gamma, \mu)$ be a non-empty TAG. The set $E(\Gamma)$ of its edges is endowed with the total order $\mu$. Say that $G$ is decomposable if it can be split into two non-empty components $G_{1}=\left(\Gamma_{1}, \mu_{1}\right)$ and $G_{2}=\left(\Gamma_{2}, \mu_{2}\right)$ such that:

1. $\mu_{i}$ is the restriction of the total order $\mu$ to $E\left(\Gamma_{i}\right), i=1,2$.

2. For any $e_{1} \in E\left(\Gamma_{1}\right)$ and $e_{2} \in E\left(\Gamma_{2}\right)$ we have $e_{1}<e_{2}$.

3. The two components are disconnected 
In that case we obviously have $G=G_{1} \cdot G_{2}$ for the product just defined above. Otherwise the TAG $G$ is called indecomposable. Iterating this decomposition process, we clearly can obtain any TAG $G$ as a finite product of indecomposable TAGs:

$$
G_{1} \cdots G_{k}
$$

with $G_{j}=\left(\Gamma_{j}, \mu_{j}\right), j=1, \ldots, k$, and where $\mu_{j}$ is the total order $\mu$ restricted to $E\left(\Gamma_{j}\right)$. The set $E\left(\Gamma_{k}\right)$ is the smallest terminal segment of $E(\Gamma)$ such that no edge in it hook to other edges in $E(\Gamma)$. Hence the last component $G_{k}$ is uniquely defined, and the whole decomposition as well by iterating this argument. Hence, the set of TAGs endowed with the product defined above is the free monoid generated by the indecomposable TAGs; this concludes the proof.

Remark 3.6 An indecomposable TAG is not necessarily connected (see Fig. 3).
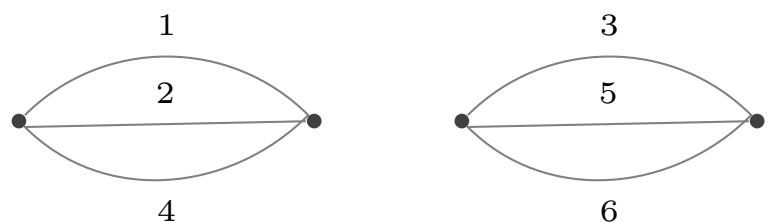

Fig. 3: A non connected indecomposable TAG.

Remark 3.7 The standard labelling of the product $\left(\Gamma_{1}, \mu_{1}\right) \cdot\left(\Gamma_{2}, \mu_{2}\right)$ is obtained by keeping the standard labelling for $E\left(\Gamma_{1}\right)$ and shifting the standard labelling of $E\left(\Gamma_{2}\right)$ by $\left|E\left(\Gamma_{1}\right)\right|$.

Let us end this section by the following example illustrating the non-commutativity of our product:

Example 3.8 One has

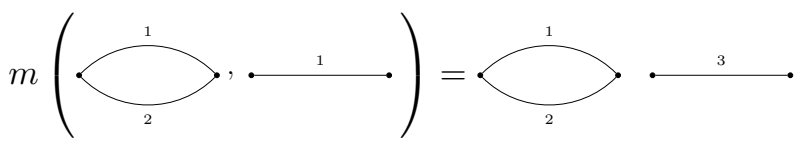

and

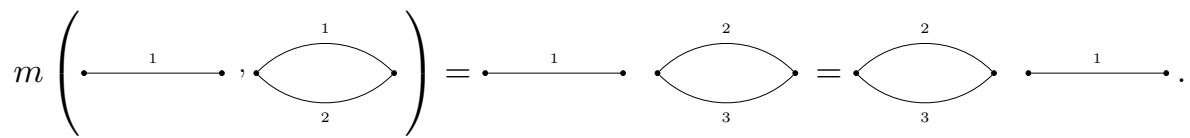

\section{Hopf algebra structure}

Let us first give the following definitions:

Definition 4.1 A subgraph $\gamma$ of a graph $\Gamma$ is the graph formed by a given subset of edges $e$ of the set of edges of the graph $\Gamma$ together with the vertices that the edges of e hook to in $\Gamma$.

Let us notice that a subgraph is not necessary connected nor spanning. 
Definition 4.2 A totally assigned subgraph $(\gamma, \nu)$ of a given TAG $(\Gamma, \mu)$ is a subgraph $\gamma$ of Tin the sense of Definition 4.1, together with the total order $\nu$ on $E(\gamma)$ induced by $\mu$. The shrinking $(\Gamma, \mu) /(\gamma, \nu)$ of a given TAG $(\Gamma, \mu)$ by a totally assigned subgraph $(\gamma, \nu)$ is defined as follows: the cograph $\Gamma / \gamma$ is obtained as usual, by shrinking each connected component of $\gamma$ on a point, and the totally ordered scale assignment $\mu / \nu$ of the cograph $\Gamma / \gamma$ is given by restricting the total order $\mu$ on the edges of the cograph, i.e. the edges of $\Gamma$ which are not internal to $\gamma$. The TAG $(\Gamma / \gamma, \mu / \nu)$ is called a totally assigned cograph.

Let us now define the coproduct $\Delta: \mathcal{H} \longrightarrow \mathcal{H} \otimes \mathcal{H}$ as

$$
\Delta((\Gamma, \mu))=\sum_{\emptyset \subseteq(\gamma, \nu) \subseteq(\Gamma, \mu)}(\gamma, \nu) \otimes(\Gamma / \gamma, \mu / \nu)
$$

for any TAG $(\Gamma, \mu)$.

Example 4.3 1) Let $\left(\Gamma_{1}, \mu_{1}\right)$ be the TAG in Fig. 2(a).

One has the coproduct:

$$
\Delta(G, \mu)=(G, \mu) \otimes 1_{\mathcal{H}}+1_{\mathcal{H}} \otimes(G, \mu)+2 . \quad 1 \quad \bullet \otimes
$$

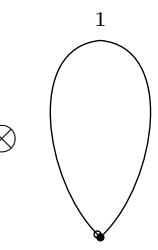

2) Let $(G, \mu)$ be the TAG given in Figure 2(b).

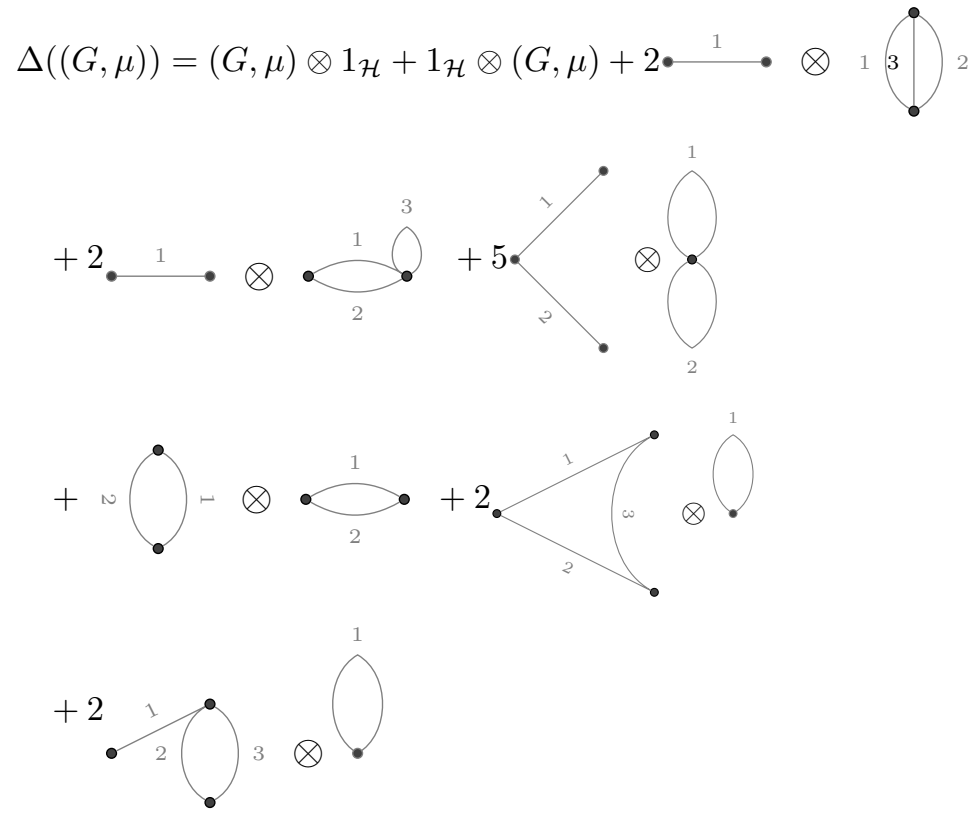


Example 4.4 Let $(G, \mu)$ be the TAG given in Fig. 4

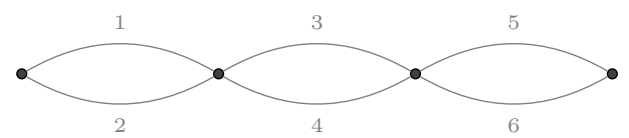

Fig. 4: Another TAG.

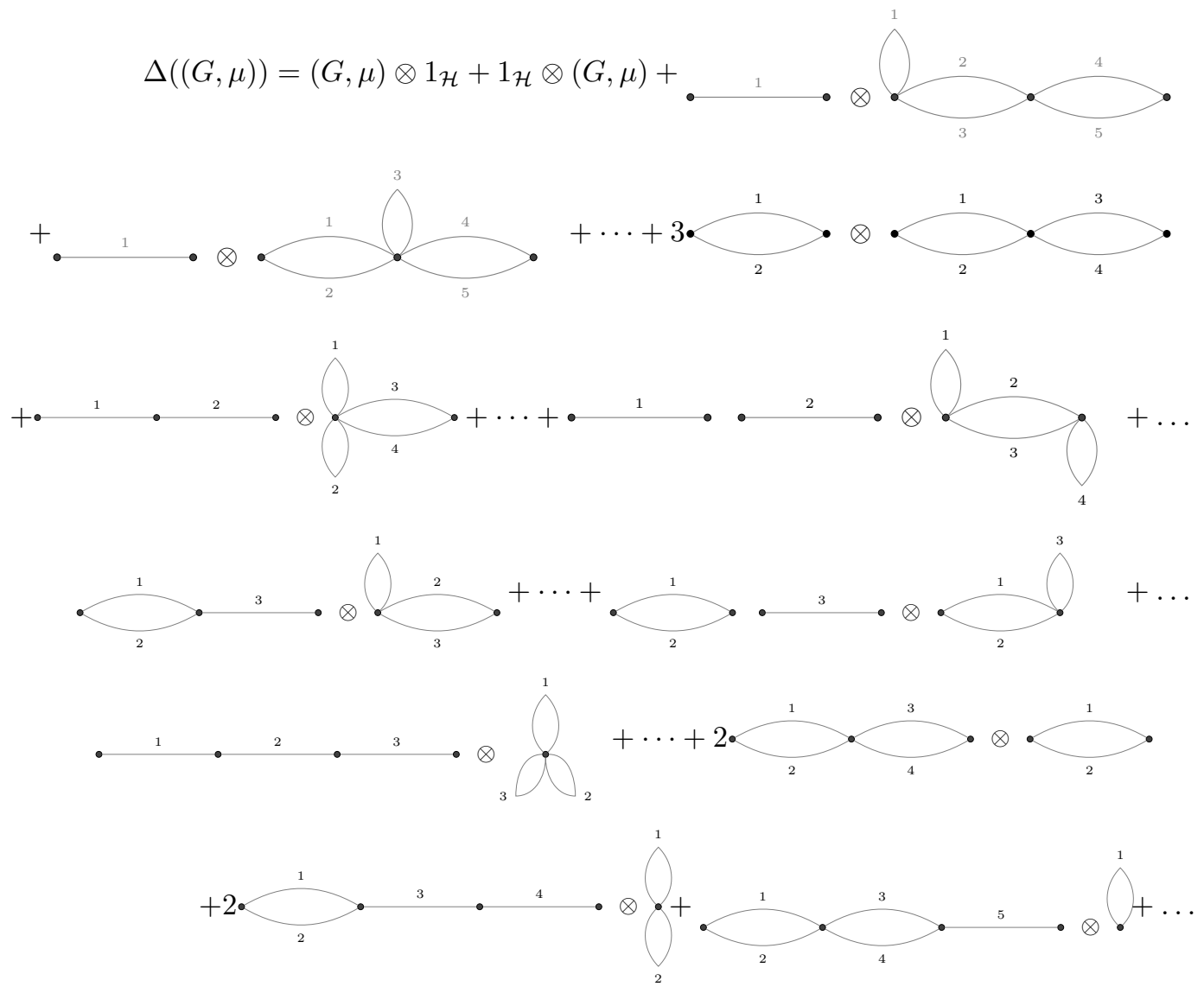

where we have denoted by ... for the similar graph obtained from choosing the same type of subgraph on the LHS on the coproduct; note that this can lead, in some cases, to distinct subgraphs on the RHS (sometimes just because of the distinct labels). 
Lemma 4.5 Let $(\Gamma, \mu)$ be a TAG in $\mathcal{H}$. Let $(\gamma, \nu)$ and $\left(\delta, \nu^{\prime}\right)$ be two totally assigned subgraphs such that $\left(\delta, \nu^{\prime}\right) \subseteq(\gamma, \nu) \subseteq(\Gamma, \mu)$. Then, one has

$$
(\Gamma / \gamma, \mu / \nu)=\left((\Gamma / \delta) /(\gamma / \delta),\left(\mu / \nu^{\prime}\right) /\left(\nu / \nu^{\prime}\right)\right)
$$

Proof: Since, $\delta \subseteq \gamma \subseteq \Gamma$, then one has $\gamma / \delta \subseteq \Gamma / \delta$. One has $\Gamma / \gamma=(\Gamma / \delta) /(\gamma / \delta)$. Moreover, the total order $\nu$ (resp. $\nu^{\prime}$ ) is induced by restriction of $\mu$ (resp. $\nu$ or $\mu$ ) to the set of edges of $\delta$. Then $\mu / \nu=\left(\mu / \nu^{\prime}\right) /\left(\nu / \nu^{\prime}\right)$, which concludes the proof.

Proposition 4.6 The coproduct defined in (6) is coassociative.

Proof: Let $(\Gamma, \mu) \in \mathcal{H}$. Then, one has:

$$
\begin{aligned}
& (\Delta \otimes I d) \circ \Delta((\Gamma, \mu))=(\Delta \otimes I d)\left(\sum_{(\gamma, \nu) \subset(\Gamma, \mu)}(\gamma, \nu) \otimes(\Gamma / \gamma, \mu / \nu)\right) \\
& =\sum_{(\gamma, \nu) \subset(\Gamma, \mu)}\left(\sum_{\left(\gamma^{\prime}, \nu^{\prime}\right) \subset(\gamma, \nu)}\left(\gamma^{\prime}, \nu^{\prime}\right) \otimes\left(\gamma / \gamma^{\prime}, \nu / \nu^{\prime}\right)\right) \otimes(\Gamma / \gamma, \mu / \nu) \\
& =\sum_{\substack{(\gamma, \nu) \subset(\Gamma, \mu) \\
\left(\gamma^{\prime}, \nu^{\prime}\right) \subset(\gamma, \nu)}}\left(\gamma^{\prime}, \nu^{\prime}\right) \otimes\left(\gamma / \gamma^{\prime}, \nu / \nu^{\prime}\right) \otimes(\Gamma / \gamma, \mu / \nu) .
\end{aligned}
$$

and

$$
\begin{aligned}
& (I d \otimes \Delta) \circ \Delta((\Gamma, \mu))=(I d \otimes \Delta)\left(\sum_{(\gamma, \nu)) \subset(\Gamma, \mu)}(\gamma, \nu) \otimes(\Gamma / \gamma, \mu / \nu)\right) \\
& =\sum_{(\gamma, \nu) \subset(\Gamma, \mu)}(\gamma, \nu) \otimes\left(\sum_{\left(\gamma^{\prime}, \nu^{\prime}\right) \subset(\Gamma / \gamma, \mu / \nu)}\left(\gamma^{\prime}, \nu^{\prime}\right) \otimes\left((\Gamma / \gamma) / \gamma^{\prime},(\mu / \nu) / \nu^{\prime}\right)\right) .
\end{aligned}
$$

There is a one-to-one correspondence between the assigned subgraphs $\left(\gamma^{\prime}, \nu^{\prime}\right) \subseteq(\Gamma / \gamma, \mu / \nu)$ and the assigned subgraphs $\left(\gamma_{1}, \nu_{1}\right) \subseteq(\Gamma, \mu)$ such that $(\gamma, \nu) \subseteq\left(\gamma_{1}, \nu_{1}\right)$. Indeed, starting from an assigned subgraph $\left(\gamma_{1}, \nu_{1}\right) \subseteq(\Gamma, \mu)$ such that $(\gamma, \nu) \subseteq\left(\gamma_{1}, \nu_{1}\right)$, we find an assigned subgraph $\left(\gamma^{\prime}, \nu^{\prime}\right) \subseteq(\Gamma / \gamma, \mu / \nu)$ by restricting the total order $\nu_{1}$ to the edges of $\gamma_{1}$ which are not internal to $\gamma$, and the inverse operation consists in extending the total order $\nu^{\prime}$ to all edges of $\gamma_{1}$ in the unique way compatible with the total order $\mu$ on $E(\Gamma)$.

Applying Lemma 4.5, one has $\left((\Gamma / \gamma) / \gamma^{\prime},(\mu / \nu) / \nu^{\prime}\right)=\left(\Gamma / \gamma_{1}, \mu / \nu_{1}\right)$. Equation (9) can then be rewritten as follows:

$$
\begin{aligned}
& (I d \otimes \Delta) \circ \Delta((\Gamma, \mu)) \\
& =\sum_{\substack{\left(\gamma_{1}, \nu_{1}\right) \subset(\Gamma, \mu) \\
(\gamma, \nu) \subset\left(\gamma_{1}, \nu_{1}\right)}}(\gamma, \nu) \otimes\left(\gamma_{1} / \gamma, \nu_{1} / \nu\right) \otimes\left(\Gamma / \gamma_{1}, \mu / \nu_{1}\right) .
\end{aligned}
$$




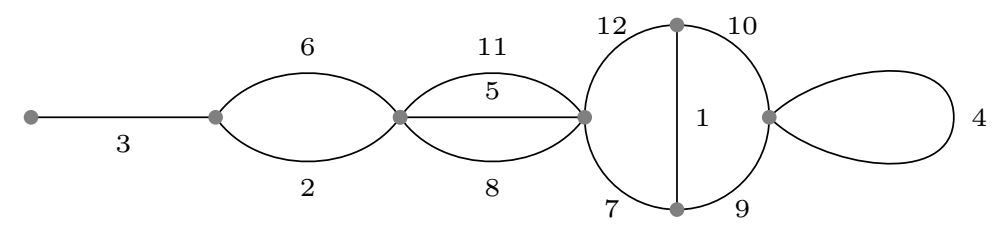

Fig. 5: A standard labeled TAG.
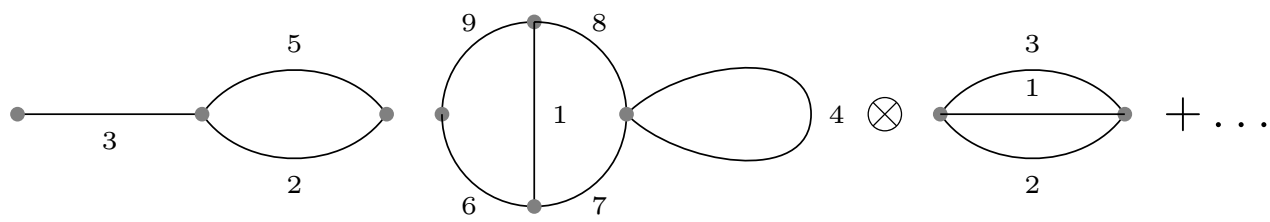

Fig. 6: One of the terms obtained by acting with the coproduct on the standard labeled TAG of Fig 5

Using equations $(8)$ and $(10)$, one concludes the proof.

Furthermore, we define the counit $\epsilon: \mathcal{H} \longrightarrow \mathbb{K}$ by

$$
\epsilon((\Gamma, \mu))=\left\{\begin{array}{l}
1 \text { if }(\Gamma, \mu)=1_{\mathcal{H}} \\
0 \text { otherwise }
\end{array}\right.
$$

Theorem 4.7 The triple $(\mathcal{H}, \Delta, \epsilon)$ is a coassociative coalgebra with counit.

Proof: Let us show that $\epsilon$ is a counit of the coalgebra. For any TAG $(\Gamma, \mu)$, one has

$$
(\epsilon \otimes I d) \circ \Delta((\Gamma, \mu))=\epsilon((\Gamma, \mu)) 1+\epsilon\left(1_{\mathcal{H}}\right)(\Gamma, \mu)+\sum_{(\gamma, \nu) \subsetneq(\Gamma, \mu)} \epsilon(\gamma, \nu)(\Gamma / \gamma, \mu / \nu)=(\Gamma, \mu) .
$$

Analogously, one has: $(I d \otimes \epsilon) \circ \Delta((\Gamma, \mu))=(\Gamma, \mu)$. One thus concludes that the maps $I d,(\epsilon \otimes I d) \circ \Delta$ and $(I d \otimes \epsilon) \circ \Delta$ coincide on TAGs, thus proving that $\epsilon$ is a counit of $\Delta$. Using now Proposition 4.6, one concludes the proof.

Example 4.8 Let us check the coassociativity of our coproduct on the example of the standard labeled TAG of Fig. 5. When acting with the coproduct on this standard labeled TAG, one gets the term of Fig. 6. which obviously adds up to the rest of the coproduct terms. Another type of term is the one of Fig. 7 (which again adds up to the rest of the coproduct terms). Acting now on these terms with $(\Delta \otimes I d)$ and respectively with $(I d \otimes \Delta)$ leads to the same term represented in Fig. 8 with standard labelling. Let us also emphasize that this term cannot be obtained from other terms of $\Delta$ because of the diagrammatic difference of the various disconnected components of the graphs. 


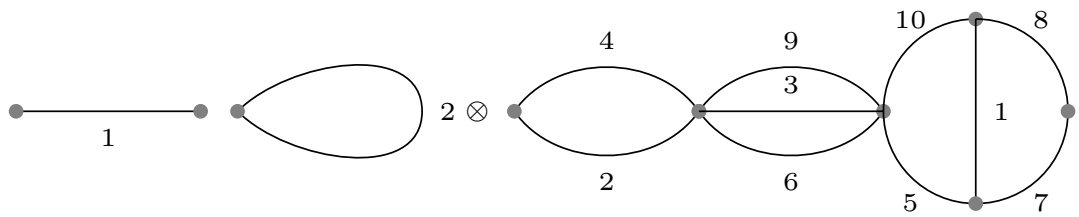

Fig. 7: Another terms obtained by acting with the coproduct on the standard labeled TAG of Fig 5
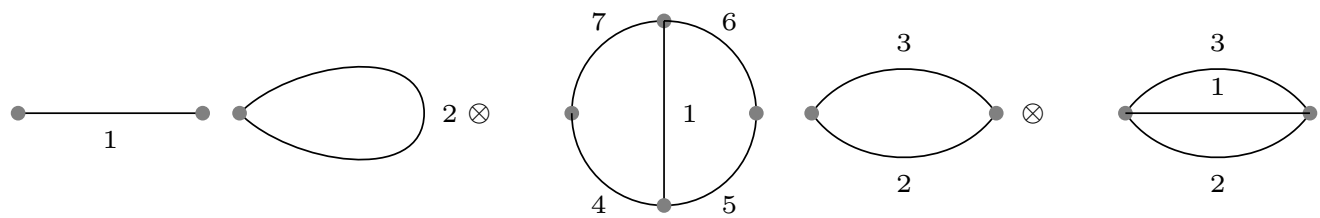

Fig. 8: The resulting in the LHS and RHS of the coassociativity identity.

One has:

Proposition 4.9 Let $\left(\Gamma_{1}, \mu_{1}\right)$ and $\left(\Gamma_{2}, \mu_{2}\right)$ be two TAGs in $\mathcal{H}$. One has

$$
\Delta\left(m\left(\left(\Gamma_{1}, \mu_{1}\right),\left(\Gamma_{2}, \mu_{2}\right)\right)\right)=m^{\otimes 2} \circ \tau_{23}\left(\Delta\left(\Gamma_{1}, \mu_{1}\right), \Delta\left(\Gamma_{2}, \mu_{2}\right)\right)
$$

where $\tau_{23}$ is the flip of the two middle factors in $\mathcal{H}^{\otimes \triangle}$.

Proof: One has

$$
\begin{aligned}
\Delta\left(m\left(\left(\Gamma_{1}, \mu_{1}\right),\left(\Gamma_{2}, \mu_{2}\right)\right)\right)= & \Delta\left(\Gamma_{1} \sqcup \Gamma_{2}, \mu_{1} \sqcup \mu_{2}\right) \\
= & \sum_{\emptyset \subseteq(g, \nu) \subseteq\left(\Gamma_{1} \sqcup \Gamma_{2}, \mu_{1} \sqcup \mu_{2}\right)}(\gamma, \nu) \otimes\left(\left(\Gamma_{1} \sqcup \Gamma_{2}\right) / \gamma,\left(\mu_{1} \sqcup \mu_{2}\right) / \nu\right) \\
= & \sum_{\substack{\left(\gamma_{1}, \nu_{1}\right) \subseteq\left(\Gamma_{1}, \mu_{1}\right) \\
\left(\gamma_{2}, \nu_{2}\right) \subseteq\left(\Gamma_{2}, \mu_{2}\right)}}\left(\gamma_{1} \sqcup \gamma_{2}, \nu_{1} \sqcup \nu_{2}\right) \otimes\left(\Gamma_{1} / \gamma_{1} \sqcup \Gamma_{2} / \gamma_{2}, \mu_{1} / \nu_{1} \sqcup \mu_{2} / \nu_{2}\right) \\
= & m^{\otimes 2} \circ \tau_{23}\left(\Delta\left(\Gamma_{1}, \mu_{1}\right), \Delta\left(\Gamma_{2}, \mu_{2}\right)\right)
\end{aligned}
$$

Example 4.10 Let $\left(\Gamma_{1}, \mu_{1}\right)$ and $\left(\Gamma_{2}, \mu_{2}\right)$ be the graph in Fig. $2(a)$. 
One has:

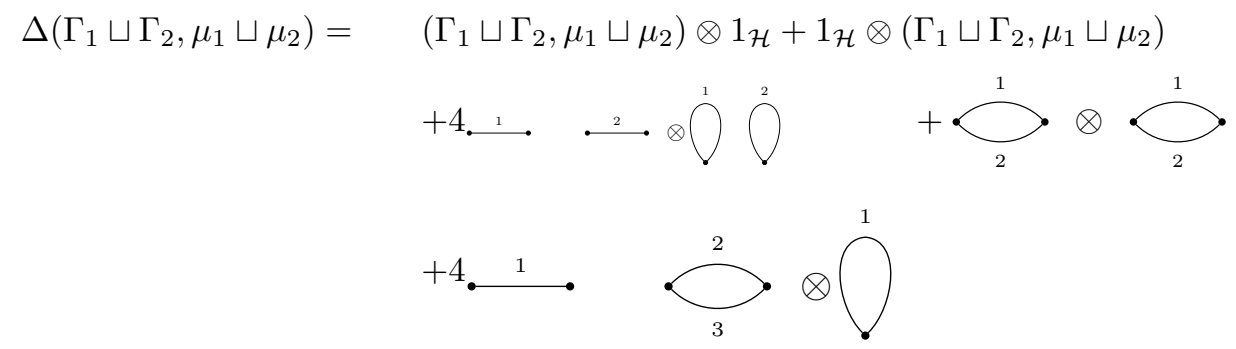

One has:

Theorem $4.11\left(\mathcal{H}, m, 1_{\mathcal{H}}, \Delta, \epsilon\right)$ is a bialgebra.

Proof: Using Proposition 4.9 it follows that $\Delta$ is a morphism of algebras. One thus concludes the proof.

For all $n \in \mathbb{N}$, one calls $\mathcal{H}(n)$ the vector space generated by the TAGs with $n$ edges. Then one has $\mathcal{H}=\bigoplus_{n \in \mathbb{N}} \mathcal{H}(n)$. Moreover, one has:

1. For all $m, n \in \mathbb{N}, \mathcal{H}(m) \mathcal{H}(n) \subseteq \mathcal{H}(m+n)$.

2. For all $n \in \mathbb{N}, \Delta(\mathcal{H}(n)) \subseteq \sum_{i+j=n} \mathcal{H}(i) \otimes \mathcal{H}(j)$.

One thus concludes that $\mathcal{H}$ is a graded bialgebra. Note that $\mathcal{H}$ is connected.

We can now state the main result of this section:

Theorem 4.12 The bialgebra $\left(\mathcal{H}, m, 1_{\mathcal{H}}, \Delta, \epsilon\right)$ is a Hopf algebra.

Proof: The bialgebra $\left(\mathcal{H}, m, 1_{\mathcal{H}}, \Delta, \epsilon\right)$ is connected and graded. The conclusion follows. The antipode $S: \mathcal{H} \longrightarrow \mathcal{H}$ verifies $S\left(1_{\mathcal{H}}\right)=1_{\mathcal{H}}$, and is given on a non-empty TAG $(\Gamma, \mu)$ by one of the two following recursive formulas:

$$
\begin{aligned}
S(\Gamma, \mu) & =-(\Gamma, \mu)-\sum_{\emptyset \subsetneq(\gamma, \nu) \subsetneq(\Gamma, \mu)} S(\gamma, \nu) \cdot(\Gamma / \gamma, \mu / \nu) \\
& =-(\Gamma, \mu)-\sum_{\emptyset \subsetneq(\gamma, \nu) \subsetneq(\Gamma, \mu)}(\gamma, \nu) \cdot S(\Gamma / \gamma, \mu / \nu) .
\end{aligned}
$$

Note that if one considers now the Hopf algebra $\mathcal{H}^{c}$ of graphs (without any edge scale decoration), one has:

Proposition 4.13 The map $\pi$ from $\mathcal{H}$ to $\mathcal{H}^{c}$ defined on the TAGs by $\pi((\Gamma, \mu))=\Gamma$ is a Hopf algebra morphism.

Proof: This statement directly follows from the definitions. 


\section{Quadri-coalgebra structure}

A quadri-algebra ([1]) is a family $(A, \nwarrow, \swarrow, \searrow, \nearrow)$, such that $A$ is a vector space, and $\nwarrow, \swarrow, \searrow, \nearrow$ are four products on $A$, satisfying nine axioms. Putting $\leftarrow=\nwarrow+\swarrow, \rightarrow=\nearrow+\searrow, \uparrow=\nwarrow+\nearrow, \downarrow=\swarrow+\searrow$, and $\star=\nwarrow+\swarrow+\searrow+\nearrow=\leftarrow+\rightarrow=\uparrow+\downarrow$, these axioms imply that $(A, \uparrow, \downarrow)$ and $(A, \leftarrow, \rightarrow)$ are dendriform algebras $([23,24])$, and $\star$ is an associative (non unitary) product. Dually, a quadri-coalgebra is a family $\left(C, \Delta_{\nwarrow}, \Delta_{\swarrow}, \Delta_{\searrow}, \Delta_{\nearrow}\right)$, where $C$ is a vector space, $, \Delta_{\nwarrow}, \Delta_{\swarrow}, \Delta_{\searrow}, \Delta_{\nearrow}: C \longrightarrow C \otimes C$, such that:

$$
\begin{aligned}
\left(\Delta_{\nwarrow} \otimes I d\right) \circ \Delta_{\nwarrow} & =\left(I d \otimes \Delta_{\star}\right) \circ \Delta_{\nwarrow}, & \left(\Delta_{\nearrow} \otimes I d\right) \circ \Delta_{\nwarrow} & =\left(I d \otimes \Delta_{\leftarrow}\right) \circ \Delta_{\nearrow}, \\
\left(\Delta_{\uparrow} \otimes I d\right) \circ \Delta_{\nearrow} & =\left(I d \otimes \Delta_{\rightarrow}\right) \circ \Delta_{\nearrow}, & \left(\Delta_{\swarrow} \otimes I d\right) \circ \Delta_{\nwarrow} & =\left(I d \otimes \Delta_{\uparrow}\right) \circ \Delta_{\swarrow}, \\
\left(\Delta_{\searrow} \otimes I d\right) \circ \Delta_{\nwarrow} & =\left(I d \otimes \Delta_{\nwarrow}\right) \circ \Delta_{\searrow}, & \left(\Delta_{\downarrow} \otimes I d\right) \circ \Delta_{\nearrow} & =\left(I d \otimes \Delta_{\nearrow}\right) \circ \Delta_{\searrow}, \\
\left(\Delta_{\leftarrow} \otimes I d\right) \circ \Delta_{\swarrow} & =\left(I d \otimes \Delta_{\downarrow}\right) \circ \Delta_{\swarrow}, & \left(\Delta_{\rightarrow} \otimes I d\right) \circ \Delta_{\swarrow} & =\left(I d \otimes \Delta_{\swarrow}\right) \circ \Delta_{\searrow}, \\
\left(\Delta_{\star} \otimes I d\right) \circ \Delta_{\searrow} & =\left(I d \otimes \Delta_{\searrow}\right) \circ \Delta_{\searrow}, & &
\end{aligned}
$$

where $\Delta_{\leftarrow}=\Delta_{\nwarrow}+\Delta_{\swarrow}, \Delta_{\rightarrow}=\Delta_{\searrow}+\Delta_{\nearrow}, \Delta_{\uparrow}=\Delta_{\nwarrow}+\Delta_{\nearrow}, \Delta_{\downarrow}=\Delta_{\swarrow}+\Delta_{\searrow}$ and $\Delta_{\star}=$ $\Delta_{\nwarrow}+\Delta_{\swarrow}+\Delta_{\searrow}+\Delta_{\nearrow}$. This implies that $\left(C, \Delta_{\leftarrow}, \Delta_{\rightarrow}\right)$ and $\left(C, \Delta_{\uparrow}, \Delta_{\downarrow}\right)$ are dendriform coalgebras; moreover, $\Delta_{\star}$ is coassociative (non counitary): it is called the coassociative coproduct induced by the quadri-coalgebra structure.

Definition 5.1 1. Let $\mathcal{H}_{+}$be the augmentation ideal of $\mathcal{H}$. It is given a coassociative, non counitary coprodut $\Delta_{\star}$ defined for all nonempty TAG $(\Gamma, \mu)$ by:

$$
\Delta_{\star}((\Gamma, \mu))=\Delta((\Gamma, \mu))-(\Gamma, \mu) \otimes 1-1 \otimes(\Gamma, \mu)=\sum_{\emptyset \subsetneq(\gamma, \nu) \subsetneq(\Gamma, \mu)}(\gamma, \nu) \otimes(\Gamma / \gamma, \mu / \nu),
$$

2. Let $(\Gamma, \mu)$ be a nonempty TAG. We denote by a $(\Gamma, \mu)$ the smallest edge of $(\Gamma, \mu)$ and $b(\Gamma, \mu)$ the greatest edge of $(\Gamma, \mu)$ for the scale assignment $\mu$.

Proposition 5.2 We define four coproducts on $\mathcal{H}_{+}$by:

$$
\begin{aligned}
& \Delta_{\nwarrow}((G, \mu))=\sum_{\substack{\emptyset \subsetneq(\gamma, \nu) \subsetneq(\Gamma, \mu), a(G) \in \gamma, b(G) \in \gamma}}(\gamma, \nu) \otimes(\Gamma / \gamma, \mu / \nu), \\
& \Delta_{\swarrow}((G, \mu))=\sum_{\substack{\emptyset \subsetneq(\gamma, \nu) \subsetneq(\Gamma, \mu), a(G) \in \gamma, b(G) \notin \gamma}}(\gamma, \nu) \otimes(\Gamma / \gamma, \mu / \nu), \\
& \Delta_{\searrow}((G, \mu))=\sum_{\substack{\emptyset \subsetneq(\gamma, \nu) \subsetneq(\Gamma, \mu), a(G) \notin \gamma, b(G) \notin \gamma}}(\gamma, \nu) \otimes(\Gamma / \gamma, \mu / \nu), \\
& \Delta_{\nearrow}((G, \mu))=\sum_{\substack{\emptyset \subsetneq(\gamma, \nu) \subsetneq(\Gamma, \mu), a(G) \notin \gamma, b(G) \in \gamma}}(\gamma, \nu) \otimes(\Gamma / \gamma, \mu / \nu) .
\end{aligned}
$$

Then $\left(\mathcal{H}_{+}, \Delta_{\nwarrow}, \Delta_{\swarrow}, \Delta_{\searrow}, \Delta_{\nearrow}\right)$ is a quadri-coalgebra, and the induced coassociative coproduct is $\Delta_{\star}$. 
Moreover, for all $x, y \in \mathcal{H}_{+}$:

$$
\begin{aligned}
& \Delta_{\nwarrow}(x y)=x y_{\uparrow}^{\prime} \otimes y_{\uparrow}^{\prime \prime}+x_{\leftarrow}^{\prime} y \otimes x_{\leftarrow}^{\prime \prime}+x_{\leftarrow}^{\prime} y_{\uparrow}^{\prime} \otimes x_{\leftarrow}^{\prime \prime} y_{\uparrow}^{\prime \prime} \\
& \Delta_{\swarrow}(x y)=x \otimes y+x_{\leftarrow}^{\prime} \otimes x_{\leftarrow}^{\prime \prime} y+x y_{\downarrow}^{\prime} \otimes y_{\downarrow}^{\prime \prime}+x_{\leftarrow}^{\prime} y_{\downarrow}^{\prime} \otimes x_{\leftarrow}^{\prime \prime} y_{\downarrow}^{\prime \prime} \\
& \Delta_{\searrow}(x y)=x_{\rightarrow}^{\prime} \otimes x_{\rightarrow}^{\prime \prime} y+y_{\downarrow}^{\prime} \otimes x y_{\downarrow}^{\prime \prime}+x_{\rightarrow}^{\prime} y_{\downarrow}^{\prime} \otimes x_{\rightarrow}^{\prime \prime} y_{\downarrow}^{\prime \prime} \\
& \Delta_{\nearrow}(x y)=y \otimes x+y_{\uparrow}^{\prime} \otimes x y_{\uparrow}^{\prime \prime}+x_{\rightarrow}^{\prime} y \otimes x_{\rightarrow}^{\prime \prime}+x_{\rightarrow}^{\prime} y_{\downarrow}^{\prime} \otimes x_{\rightarrow}^{\prime \prime} y_{\downarrow}^{\prime \prime},
\end{aligned}
$$

where $\Delta_{\leftarrow}(x)=x_{\leftarrow}^{\prime} \otimes x_{\leftarrow}^{\prime \prime}, \Delta_{\rightarrow}(x)=x_{\rightarrow}^{\prime} \otimes x_{\rightarrow}^{\prime \prime}, \Delta_{\uparrow}(y)=y_{\uparrow}^{\prime} \otimes y_{\uparrow}^{\prime \prime}$, and $\Delta_{\downarrow}(y)=y_{\downarrow}^{\prime} \otimes y_{\downarrow}^{\prime \prime}$.

Proof: Obviously, $\Delta_{\nwarrow}+\Delta_{\swarrow}+\Delta_{\searrow}+\Delta_{\nearrow}=\Delta_{\star}$. Let $(G, \mu)$ be a nonempty TAG. We write, using the coassociativity of $\Delta_{\star}$ :

$$
=\begin{gathered}
\left(\Delta_{\star} \otimes I d\right) \circ \Delta_{\star}((\Gamma, \mu))=\left(I d \otimes \Delta_{\star}\right) \circ \Delta_{\star}((\Gamma, \mu)) \\
\sum_{\emptyset \subsetneq\left(\gamma^{\prime}, \nu^{\prime}\right) \subsetneq(\gamma, \nu) \subsetneq(\Gamma, \mu)}\left(\gamma^{\prime}, \nu^{\prime}\right) \otimes\left(\gamma / \gamma^{\prime}, \nu / \nu^{\prime}\right) \otimes(\Gamma / \gamma, \mu / \nu) .
\end{gathered}
$$

Then each relation defining quadri-coalgebras corresponds to the terms in this sum such that:

$$
\begin{array}{cr}
a \in \gamma^{\prime}, b \in \gamma^{\prime}, & a \in \gamma / \gamma^{\prime}, b \in \gamma^{\prime}, \\
a \in \Gamma / \gamma, b \in \gamma^{\prime}, & a \in \gamma^{\prime}, b \in \gamma / \gamma^{\prime}, \\
a \in \gamma / \gamma^{\prime}, b \in \gamma / \gamma^{\prime}, & a \in \Gamma / \gamma, b \in \gamma / \gamma^{\prime}, \\
a \in \gamma^{\prime}, b \in \Gamma / \gamma, & a \in \gamma / \gamma^{\prime}, b \in \Gamma / \gamma, \\
a \in \Gamma / \gamma, b \in \Gamma / \gamma . &
\end{array}
$$

Let $x, y$ be nonempty TAGs. By definition of the product, $a(x y)=a(x)$ and $b(x y)=b(y)$. Let $\emptyset \subsetneq$ $(\gamma, \nu) \subsetneq x y$. We put $\left(\gamma_{x}, \nu_{x}\right)=x \cap(\gamma, \nu)$ and $\left(\gamma_{y}, \nu_{y}\right)=y \cap(\gamma, \nu)$. Then $(\gamma, \nu)=\left(\gamma_{x}, \nu_{x}\right)\left(\gamma_{y}, \nu_{y}\right)$ and $x y /(\gamma, \nu)=x /\left(\gamma_{x}, \nu_{x}\right) y /\left(\gamma_{y}, \nu_{y}\right)$. If $a(x, y) \in \gamma, b(x y) \notin \gamma$, then $a(x) \in \gamma_{x}$ and $a(y) \notin \gamma_{y}$. Four cases are possible:

- $x=\gamma$. This gives the term $x \otimes y$.

- $\gamma \subsetneq x$. This gives the term $x_{\leftarrow}^{\prime} \otimes x_{\leftarrow}^{\prime \prime} y$.

- $x \subsetneq \gamma$. This gives the term $x y_{\downarrow}^{\prime} \otimes y_{\downarrow}^{\prime \prime}$.

- $\gamma_{x}, \gamma_{y} \neq \emptyset$ and $\gamma_{x} \subsetneq x, \gamma_{y} \subsetneq y$. This gives the term $x_{\leftarrow}^{\prime} y_{\downarrow}^{\prime} \otimes x_{\leftarrow}^{\prime \prime} y_{\downarrow}^{\prime \prime}$.

This proves the compatibility between $\Delta_{\swarrow}$ and the product. The three other compatibilities are proved in the same way.

Summing, we obtain the following compatibilities:

$$
\begin{aligned}
\Delta_{\leftarrow}(x y) & =x \otimes y+x y^{\prime} \otimes y^{\prime \prime}+x_{\leftarrow}^{\prime} y \otimes x_{\leftarrow}^{\prime \prime}+x_{\leftarrow}^{\prime} \otimes x_{\leftarrow}^{\prime \prime} y+x_{\leftarrow}^{\prime} y^{\prime} \otimes x_{\leftarrow}^{\prime \prime} y^{\prime \prime} \\
\Delta_{\rightarrow}(x y) & =y \otimes x+y^{\prime} \otimes x y^{\prime \prime}+x_{\rightarrow}^{\prime} \otimes x_{\rightarrow}^{\prime \prime} y+x_{\rightarrow}^{\prime} y \otimes x_{\rightarrow}^{\prime \prime}+x_{\rightarrow}^{\prime} y^{\prime} \otimes x_{\rightarrow}^{\prime \prime} y^{\prime \prime} \\
\Delta_{\uparrow}(x y) & =y \otimes x+x^{\prime} y \otimes x^{\prime \prime}+x y_{\uparrow}^{\prime} \otimes y_{\uparrow}^{\prime \prime}+y_{\uparrow}^{\prime} \otimes x y_{\uparrow}^{\prime \prime}+x^{\prime} y_{\uparrow}^{\prime} \otimes x^{\prime \prime} y_{\uparrow}^{\prime \prime} \\
\Delta_{\downarrow}(x y) & =x \otimes y+x^{\prime} \otimes x^{\prime \prime} y+x y_{\downarrow}^{\prime} \otimes y_{\downarrow}^{\prime \prime}+y_{\downarrow}^{\prime} \otimes x y_{\downarrow}^{\prime \prime}+x^{\prime} y_{\downarrow}^{\prime} \otimes x^{\prime \prime} y_{\downarrow}^{\prime \prime} .
\end{aligned}
$$

Hence: 
Corollary $5.3\left(\mathcal{H}_{+}, m^{o p}, \Delta_{\leftarrow}, \Delta_{\rightarrow}\right)$ and $\left(\mathcal{H}_{+}, m, \Delta_{\uparrow}, \Delta_{\downarrow}\right)$ are condendriform Hopf algebras ([[14]).

Let us end this paper by the following concluding remarks. A further non-commutative Hopf algebra of TAGs can be defined when considering only graphs of a given quantum field theoretical model and defining the coproduct as the appropriate sum on the class of superficially divergent graphs (see for example [20], where such a Hopf algebra was defined, in a commutative setting).

The non-commutative graph Hopf algebraic structure defined here is a combinatorial Hopf algebra (CHA) using a selection/contraction coproduct rule - this type of CHAs being called CHAs of type I in [32]. Examples of such CHAs are the Connes-Kreimer Hopf algebras of quantum field theory [7, 8, 9], of non-commutative Moyal quantum field theory [34, 35] (see also [33] for a short review), of quantum gravity spin-foam models [26, 31], of random tensor models [27], or the word Hopf algebra WMat [10]. This type of coproduct rule is fundamentally different of the selection/deletion rule used in CHAs such as FQSym, MQSym, the Loday-Ronco Hopf algebra etc. - CHAs of type II (see again [32]). It seems however interesting to us to investigate in what circumstances one can find some non-trivial mathematical relations between these two types of CHAs.

\section{Acknowledgements}

G. H. E. Duchamp, N. Hoang-Nghia and A. Tanasa are partially supported by the "Combinatoire algébrique" Univ. Paris 13, Sorbonne Paris Cité BQR grant. A. Tanasa further acknowledges the grant PN 093701 02 .

\section{References}

[1] M. Aguiar and J.-L. Loday. Quadri-algebras. Journal of Pure and Applied Algebra, 3:205-221, 2004.

[2] G. Benfatto and G. Gallavotti. Perturbation theory of the Fermi surface in a quantum liquid. A general quasi-particle formalism and one dimensional systems. Journ. Stat. Physics, 59(541), 1990.

[3] O. Boruvka. On a minimal problem. Práce Moravské Pridovedecké Spolecnosti, 3, 1926.

[4] C. Brouder. Runge-Kutta methods and renormalization. Eur. Phys. J., 12:521-534, 2000.

[5] J. C. Butcher. An algebraic theory of integration methods. Math. Comp., 26:79-106, 1972.

[6] S. Carrozza, D. Oriti, and V. Rivasseau. Renormalization of Tensorial Group Field Theories: Abelian $\mathrm{U}(1)$ Models in Four Dimensions. arXiv:1207.6734 [hep-th].

[7] A. Connes and D. Kreimer. Hopf algebras, Renormalisation and Noncommutative Geometry. Comm. Math. Phys., 199:203-242, 1998.

[8] A. Connes and D. Kreimer. Renormalization in Quantum Field Theory and the Riemann-Hilbert problem I: the Hopf algebra structure of graphs and the main theorem. Comm. Math. Phys., 210:249$273,2000$. 
[9] A. Connes and D. Kreimer. Renormalization in Quantum Field Theory and the Riemann-Hilbert problem II: the $\beta$-function, diffeomorphisms and the renormalization group. Comm. Math. Phys., 216:215-241, 2001.

[10] G. H. E. Duchamp, N. Hoang-Nghia, and A. Tanasa. A word Hopf algebra based on the selection/quotient principle. Séminaire Lotharingien de Combinatoire, B68c, 2013. [arXiv:1207.6522 [math.CO]].

[11] A. Dür. Möbius functions, incidence algebras and power series representations, volume 1202 of Lecture Notes in Mathematics. Springer-Verlag, 1986.

[12] J. Feldman and E. Trubowitz. Perturbation Theory for Many Fermions Systems. Helv. Phys. Acta, 63(156), 1990.

[13] L. Foissy. Les algèbres de Hopf des arbres enracinés décorés, I. Bull. Sci. math., 126:193-239, 2002.

[14] L. Foissy. Bidendriform bialgebras, trees, and free quasi-symmetric functions. Journal of Pure and Applied Algebra, 2:439-459, 2007.

[15] J. B. Geloun and V. Rivasseau. A Renormalizable 4-Dimensional Tensor Field Theory. Commun. Math. Phys., 318(69), 2013. arXiv:1111.4997 [hep-th].

[16] R. Gurau, J. Magnen, V. Rivasseau, and A. Tanasa. A Translation-invariant renormalizable noncommutative scalar model. Commun. Math. Phys., 287:275-290, 2009. [arXiv:0802.0791 [math$\mathrm{ph}]]$.

[17] R. Gurau, J. Magnen, V. Rivasseau, and F. Vignes-Tourneret. Renormalization of non-commutative $\phi_{4}^{4}$ field theory in x space. Commun. Math. Phys., 267:515-542, 2006. [hep-th/0512271].

[18] R. Gurau, V. Rivasseau, and F. Vignes-Tourneret. Propagators for noncommutative field theories. Annales Henri Poincare, 7:1601-1628, 2006. [hep-th/0512071].

[19] R. Holtkamp. Comparison of Hopf algebras on trees. Archiv der Mathematik, 80:368-383, 2003.

[20] T. Krajewski, V. Rivasseau, and A. Tanasa. Combinatorial Hopf algebraic description of the multiscale renormalization in quantum field theory. Séminaire Lotharingien de Combinatoire (in press). arXiv:1211.4429.

[21] D. Kreimer. On the Hopf algebra structure of perturbative quantum field theories. Adv. Theor. Math. Phys., 2, 1998.

[22] J. B. Kruskal jr. On the shortest spanning tree of a graph and the travelling salesman problem. Proceedings of the American Mathematical Society, 7:48-50, 1956.

[23] J. L. Loday. Dialgebras in Dialgebras and related operads, pages 7-66. Lecture Notes in Math. Springer, 2008.

[24] J.-L. Loday and M. Ronco. Combinatorial Hopf Algebras. Adv. Math., 139:293-309, 1998.

[25] J. L. Loday and M. Ronco. Hopf algebra of planar binary trees. Adv. Math., 139:293-309, 1998. 
[26] F. Markopoulou. Coarse graining in spin foam models. Class. Quant. Grav., 20(777), 2003. [grqc/0203036].

[27] M. Raasakka and A. Tanasa. Combinatorial Hopf algebra for the Ben Geloun-Rivasseau tensor field theory. arXiv:1306.1022 [gr-qc], submitted.

[28] V. Rivasseau. From perturbative to constructive renormalization. Princeton University Press, 1991.

[29] V. Rivasseau. Introduction to the Renormalization Group with Applications to Non-Relativistic Quantum Electron Gases, volume 2051 of Lect. Notes Math., pages 1-54. Springer Berlin Heidelberg, 2012. [arXiv:1102.5117 [math-ph]].

[30] V. Rivasseau, F. Vignes-Tourneret, and R. Wulkenhaar. Renormalization of noncommutative phi**4theory by multi-scale analysis. Commun. Math. Phys., 262:565-594, 2006. [hep-th/0501036].

[31] A. Tanasa. Algebraic structures in quantum gravity. Class. Quant. Grav., 27(095008), 2010. [arXiv:0909.5631 [gr-qc]].

[32] A. Tanasa. Combinatorics in quantum field theory and random tensor models. Habilitation, Nov. 2012.

[33] A. Tanasa. Some combinatorial aspects of quantum field theory. Séminaire Lotharingien de Combinatoire, B65g, 2012.

[34] A. Tanasa and D. Kreimer. Combinatorial Dyson-Schwinger equations in noncommutative field theory. Journal of Noncommutative Geometry, 7(255), 2013. [arXiv:0907.2182 [hep-th]].

[35] A. Tanasa and F. Vignes-Tourneret. Hopf algebra of non-commutative field theory. Journal of Noncommutative Geometry, 2(125), 2008. [arXiv:0707.4143 [math-ph]].

[36] F. Vignes-Tourneret. Renormalization of the Orientable Non-commutative Gross-Neveu Model. Annales Henri Poincare, 8:427-474, 2007. [math-ph/0606069]. 